\title{
Functional equations for vector products and quaternions
}

\author{
Balázs Nyul and Gábor Nyul
}

\begin{abstract}
In our paper we find all functions $f: \mathbb{R} \times \mathbb{R}^{3} \rightarrow \mathbb{H}$ and $g: \mathbb{R}^{3} \rightarrow \mathbb{H}$ satisfying $f(r, \mathbf{v}) f(s, \mathbf{w})=-\langle\mathbf{v}, \mathbf{w}\rangle+f(r s, s \mathbf{v}+r \mathbf{w}+\mathbf{v} \times \mathbf{w})$ $\left(r, s \in \mathbb{R}, \mathbf{v}, \mathbf{w} \in \mathbb{R}^{3}\right)$ and $g(\mathbf{v}) g(\mathbf{w})=-\langle\mathbf{v}, \mathbf{w}\rangle+g(\mathbf{v} \times \mathbf{w})\left(\mathbf{v}, \mathbf{w} \in \mathbb{R}^{3}\right)$. These functional equations were motivated by the well-known identities for vector products and quaternions, which can be obtained from the solutions $f\left(r,\left(v_{1}, v_{2}, v_{3}\right)\right)=r+v_{1} i+v_{2} j+v_{3} k$ and $g\left(\left(v_{1}, v_{2}, v_{3}\right)\right)=$ $v_{1} i+v_{2} j+v_{3} k$.
\end{abstract}

Mathematics Subject Classification (2010). 39B52, 16K20.

Keywords. functional equation, vector product, quaternion.

\section{Quaternions and vector products}

Let $\mathbb{H}=\left\{r+v_{1} i+v_{2} j+v_{3} k \mid r, v_{1}, v_{2}, v_{3} \in \mathbb{R}\right\}$ be the skew field of quaternions with the basic relations $i^{2}=j^{2}=k^{2}=i j k=-1$. We call $r$ the real part and $v_{1} i+v_{2} j+v_{3} k$ the imaginary part of the quaternion $r+v_{1} i+v_{2} j+v_{3} k \in \mathbb{H}$. A quaternion is purely imaginary if its real part equals 0 . The absolute value of the above quaternion is defined by $\sqrt{r^{2}+v_{1}^{2}+v_{2}^{2}+v_{3}^{2}}$.

It is well-known (see e.g. [1], [6], [9], [10]) that identifying the purely imaginary quaternion $v_{1} i+v_{2} j+v_{3} k$ with the vector $\left(v_{1}, v_{2}, v_{3}\right) \in \mathbb{R}^{3}$ we have $(r+\mathbf{v})(s+\mathbf{w})=(r s-\langle\mathbf{v}, \mathbf{w}\rangle)+(s \mathbf{v}+r \mathbf{w}+\mathbf{v} \times \mathbf{w}) \quad\left(r, s \in \mathbb{R}, \mathbf{v}, \mathbf{w} \in \mathbb{R}^{3}\right)$,

and for purely imaginary quaternions

$$
\mathbf{v w}=-\langle\mathbf{v}, \mathbf{w}\rangle+\mathbf{v} \times \mathbf{w} \quad\left(\mathbf{v}, \mathbf{w} \in \mathbb{R}^{3}\right),
$$

where on the left-hand sides quaternionic products stand, $\langle\mathbf{v}, \mathbf{w}\rangle$ and $\mathbf{v} \times \mathbf{w}$ denote the standard inner product (dot product) and the cross product of $\mathbf{v}, \mathbf{w} \in \mathbb{R}^{3}$, respectively.

Research was supported in part by Grant 75566 from the Hungarian Scientific Research Fund. 
In this paper our aim is to find all such identifications between vectors and quaternions in the below sense. More precisely, we solve completely functional equations

$$
f(r, \mathbf{v}) f(s, \mathbf{w})=-\langle\mathbf{v}, \mathbf{w}\rangle+f(r s, s \mathbf{v}+r \mathbf{w}+\mathbf{v} \times \mathbf{w}) \quad\left(r, s \in \mathbb{R}, \mathbf{v}, \mathbf{w} \in \mathbb{R}^{3}\right)
$$

and

$$
g(\mathbf{v}) g(\mathbf{w})=-\langle\mathbf{v}, \mathbf{w}\rangle+g(\mathbf{v} \times \mathbf{w}) \quad\left(\mathbf{v}, \mathbf{w} \in \mathbb{R}^{3}\right)
$$

in functions $f: \mathbb{R} \times \mathbb{R}^{3} \rightarrow \mathbb{H}$ and $g: \mathbb{R}^{3} \rightarrow \mathbb{H}$.

All the solutions of (1) and (2) are given in the following theorems.

Theorem 1. The function $g: \mathbb{R}^{3} \rightarrow \mathbb{H}$ satisfies (2) if and only if

$$
\begin{aligned}
g\left(\left(v_{1}, v_{2}, v_{3}\right)\right) & =\left(a_{1} v_{1}+b_{1} v_{2}+\left(a_{2} b_{3}-a_{3} b_{2}\right) v_{3}\right) i+ \\
& +\left(a_{2} v_{1}+b_{2} v_{2}+\left(a_{3} b_{1}-a_{1} b_{3}\right) v_{3}\right) j+ \\
& +\left(a_{3} v_{1}+b_{3} v_{2}+\left(a_{1} b_{2}-a_{2} b_{1}\right) v_{3}\right) k,
\end{aligned}
$$

where $\left(a_{1}, a_{2}, a_{3}\right),\left(b_{1}, b_{2}, b_{3}\right) \in \mathbb{R}^{3}$ are orthogonal unit vectors.

Theorem 2. The function $f: \mathbb{R} \times \mathbb{R}^{3} \rightarrow \mathbb{H}$ satisfies (1) if and only if

$$
\begin{aligned}
f\left(r,\left(v_{1}, v_{2}, v_{3}\right)\right) & =r+\left(a_{1} v_{1}+b_{1} v_{2}+\left(a_{2} b_{3}-a_{3} b_{2}\right) v_{3}\right) i+ \\
& +\left(a_{2} v_{1}+b_{2} v_{2}+\left(a_{3} b_{1}-a_{1} b_{3}\right) v_{3}\right) j+ \\
& +\left(a_{3} v_{1}+b_{3} v_{2}+\left(a_{1} b_{2}-a_{2} b_{1}\right) v_{3}\right) k,
\end{aligned}
$$

where $\left(a_{1}, a_{2}, a_{3}\right),\left(b_{1}, b_{2}, b_{3}\right) \in \mathbb{R}^{3}$ are orthogonal unit vectors.

We notice that the solutions described in our theorems give the original natural identification with $a_{1}=b_{2}=1$ and $a_{2}=a_{3}=b_{1}=b_{3}=0$.

Before proving these theorems, we mention that according to our knowledge there are only a relatively few results ([2], [3], [4], [5], [7], [8]) about functional equations in connection with quaternions.

\section{Proofs}

In the proofs we will use the following lemma.

Lemma. For $h \in \mathbb{H}, h^{2}$ is a non-positive real number if and only if $h$ is purely imaginary, and in this case $h^{2}=-|h|^{2}$.

Proof. $\left(r+v_{1} i+v_{2} j+v_{3} k\right)^{2}=\left(r^{2}-v_{1}^{2}-v_{2}^{2}-v_{3}^{2}\right)+2 r v_{1} i+2 r v_{2} j+2 r v_{3} k$ is real and non-positive if and only if $r=0$.

Proof of Theorem 1. For simplicity, if we substitute $\mathbf{v}=\mathbf{v}_{0}, \mathbf{w}=\mathbf{w}_{0}$ into $(2)$, we shall say briefly that we substitute $\left(\mathbf{v}_{0}, \mathbf{w}_{0}\right)$ into $(2)\left(\mathbf{v}_{0}, \mathbf{w}_{0} \in \mathbb{R}^{3}\right)$.

Claim 1: $g(\mathbf{0})=0$.

After substitution $(\mathbf{v}, \mathbf{0})$ into $(2)$, we get $g(\mathbf{v}) g(\mathbf{0})=g(\mathbf{0})$. If $g(\mathbf{0}) \neq 0$, then multiplying this equation from the right by the multiplicative inverse of $g(\mathbf{0})$, it follows that $g(\mathbf{v})=1\left(\mathbf{v} \in \mathbb{R}^{3}\right)$. Then equation (2) would give $\langle\mathbf{v}, \mathbf{w}\rangle=0\left(\mathbf{v}, \mathbf{w} \in \mathbb{R}^{3}\right)$, which is a contradiction. It means that $g(\mathbf{0})=0$. 
Claim 2: $g(\mathbf{v})$ is purely imaginary and $|g(\mathbf{v})|=\|\mathbf{v}\|\left(\mathbf{v} \in \mathbb{R}^{3}\right)$.

Substitute $(\mathbf{v}, \mathbf{v})$ into (2). By Claim 1 we get

$$
g(\mathbf{v})^{2}=-\|\mathbf{v}\|^{2}+g(\mathbf{0})=-\|\mathbf{v}\|^{2} .
$$

Using our Lemma, it follows that $g(\mathbf{v})$ is purely imaginary and $g(\mathbf{v})^{2}=$ $-|g(\mathbf{v})|^{2}$, hence $|g(\mathbf{v})|=\|\mathbf{v}\|$.

Claim 3: $g(\lambda \mathbf{v})=\lambda g(\mathbf{v})\left(\lambda \in \mathbb{R}, \mathbf{v} \in \mathbb{R}^{3}\right)$.

Substitute $(\mathbf{v}, \lambda \mathbf{v})$ into (2). Using Claims 1 and 2 we get

$$
g(\mathbf{v}) g(\lambda \mathbf{v})=-\lambda\|\mathbf{v}\|^{2}+g(\mathbf{0})=\lambda g(\mathbf{v})^{2} .
$$

By Claim 2, $\mathbf{v} \neq \mathbf{0}$ is equivalent to $g(\mathbf{v}) \neq 0$. If $\mathbf{v} \neq \mathbf{0}$, then multiplying this equation from the left by the multiplicative inverse of $g(\mathbf{v})$ yields $g(\lambda \mathbf{v})=$ $\lambda g(\mathbf{v})$. Moreover, it follows from Claim 1 that this also holds for $\mathbf{v}=\mathbf{0}$.

Claim 4: $g(\mathbf{v}+\mathbf{w})=g(\mathbf{v})+g(\mathbf{w})\left(\mathbf{v}, \mathbf{w} \in \mathbb{R}^{3}\right)$.

Substituting $(\mathbf{v}+\mathbf{w}, \mathbf{w}),(\mathbf{v}, \mathbf{w})$ and $(\mathbf{w}, \mathbf{w})$ into $(2)$, we get the following equations:

$$
\begin{aligned}
g(\mathbf{v}+\mathbf{w}) g(\mathbf{w}) & =-\langle\mathbf{v}+\mathbf{w}, \mathbf{w}\rangle+g(\mathbf{v} \times \mathbf{w}), \\
g(\mathbf{v}) g(\mathbf{w}) & =-\langle\mathbf{v}, \mathbf{w}\rangle+g(\mathbf{v} \times \mathbf{w}), \\
g(\mathbf{w}) g(\mathbf{w}) & =-\langle\mathbf{w}, \mathbf{w}\rangle+g(\mathbf{0}) .
\end{aligned}
$$

If we subtract the second and the third equations from the first one and use Claim 1, we get

$$
(g(\mathbf{v}+\mathbf{w})-g(\mathbf{v})-g(\mathbf{w})) g(\mathbf{w})=0 .
$$

If $\mathbf{w} \neq \mathbf{0}$, then multiplying this equation from the right by the multiplicative inverse of $g(\mathbf{w})$, we have $g(\mathbf{v}+\mathbf{w})=g(\mathbf{v})+g(\mathbf{w})$, and it also holds for $\mathbf{w}=\mathbf{0}$ because of Claim 1.

Claim 5: Let $\left(\mathbf{e}_{1}, \mathbf{e}_{2}, \mathbf{e}_{3}\right)$ be the standard basis of $\mathbb{R}^{3}$. By Claim 2, $g\left(\mathbf{e}_{1}\right)$ and $g\left(\mathbf{e}_{2}\right)$ are purely imaginary. Let $g\left(\mathbf{e}_{1}\right)=a_{1} i+a_{2} j+a_{3} k, g\left(\mathbf{e}_{2}\right)=b_{1} i+b_{2} j+b_{3} k$. Then we state that $\left(a_{1}, a_{2}, a_{3}\right),\left(b_{1}, b_{2}, b_{3}\right)$ are orthogonal unit vectors.

Claim 2 also implies that $\left|g\left(\mathbf{e}_{1}\right)\right|=\left|g\left(\mathbf{e}_{2}\right)\right|=1$, hence $\left\|\left(a_{1}, a_{2}, a_{3}\right)\right\|=$ $\left\|\left(b_{1}, b_{2}, b_{3}\right)\right\|=1$.

If we substitute $\left(\mathbf{e}_{1}, \mathbf{e}_{2}\right)$ into (2), it follows that $g\left(\mathbf{e}_{1}\right) g\left(\mathbf{e}_{2}\right)=g\left(\mathbf{e}_{3}\right)$. On the other hand

$$
\begin{aligned}
g\left(\mathbf{e}_{1}\right) g\left(\mathbf{e}_{2}\right) & =\left(-a_{1} b_{1}-a_{2} b_{2}-a_{3} b_{3}\right)+ \\
& +\left(a_{2} b_{3}-a_{3} b_{2}\right) i+\left(a_{3} b_{1}-a_{1} b_{3}\right) j+\left(a_{1} b_{2}-a_{2} b_{1}\right) k .
\end{aligned}
$$

Using Claim 2, we get that $g\left(\mathbf{e}_{3}\right)$ is purely imaginary, thus $\left(a_{1}, a_{2}, a_{3}\right) \perp$ $\left(b_{1}, b_{2}, b_{3}\right)$ and $g\left(\mathbf{e}_{3}\right)=\left(a_{2} b_{3}-a_{3} b_{2}\right) i+\left(a_{3} b_{1}-a_{1} b_{3}\right) j+\left(a_{1} b_{2}-a_{2} b_{1}\right) k$.

Claim 6: The solutions of (2) are precisely the functions given in the theorem.

By linearity, $g\left(\left(v_{1}, v_{2}, v_{3}\right)\right)=v_{1} g\left(\mathbf{e}_{1}\right)+v_{2} g\left(\mathbf{e}_{2}\right)+v_{3} g\left(\mathbf{e}_{3}\right)$. From Claim 5 we get the formula given in the theorem.

It can be checked by direct calculation that these are indeed solutions of $(2)$.

In the resolution of equation (1) we will use Theorem 1. 
Proof of Theorem 2. Similarly to the above proof, if we substitute $r=r_{0}$, $\mathbf{v}=\mathbf{v}_{0}, s=s_{0}, \mathbf{w}=\mathbf{w}_{0}$ into (1), we shall say briefly that we substitute $\left(r_{0}, \mathbf{v}_{0}, s_{0}, \mathbf{w}_{0}\right)$ into $(1)\left(r_{0}, s_{0} \in \mathbb{R}, \mathbf{v}_{0}, \mathbf{w}_{0} \in \mathbb{R}^{3}\right)$.

Let $g(\mathbf{v})=f(0, \mathbf{v})\left(\mathbf{v} \in \mathbb{R}^{3}\right)$.

Claim 1: $g$ is linear, $g(\mathbf{v})=0$ if and only if $\mathbf{v}=\mathbf{0}$, and $g$ can be given as in Theorem 1.

By substituting $(0, \mathbf{v}, 0, \mathbf{w})$ into $(1)$, we get

$$
f(0, \mathbf{v}) f(0, \mathbf{w})=-\langle\mathbf{v}, \mathbf{w}\rangle+f(0, \mathbf{v} \times \mathbf{w}),
$$

which means that $g$ satisfies (2). Then our statements follow from Theorem 1 and its proof.

Claim 2: $f(r, \mathbf{0})=r(r \in \mathbb{R})$.

Substitute $(r, \mathbf{0}, 0, \mathbf{w})$ into (1). From Claim 1 it follows that

$$
f(r, \mathbf{0}) f(0, \mathbf{w})=f(0, r \mathbf{w})=r f(0, \mathbf{w}) .
$$

If $\mathbf{w} \neq \mathbf{0}$, then we can multiply this equation from the right by the multiplicative inverse of $f(0, \mathbf{w})$ and get $f(r, \mathbf{0})=r$.

Claim 3: $f(r, \mathbf{v})=f(r, \mathbf{0})+f(0, \mathbf{v})\left(r \in \mathbb{R}, \mathbf{v} \in \mathbb{R}^{3}\right)$.

Substitute $(r, \mathbf{v}, 0, \mathbf{v}),(r, \mathbf{0}, 0, \mathbf{v})$ and $(0, \mathbf{v}, 0, \mathbf{v})$ into (1) to obtain the following equations:

$$
\begin{aligned}
& f(r, \mathbf{v}) f(0, \mathbf{v})=-\langle\mathbf{v}, \mathbf{v}\rangle+f(0, r \mathbf{v}), \\
& f(r, \mathbf{0}) f(0, \mathbf{v})=f(0, r \mathbf{v}), \\
& f(0, \mathbf{v}) f(0, \mathbf{v})=-\langle\mathbf{v}, \mathbf{v}\rangle+f(0, \mathbf{0}) .
\end{aligned}
$$

Subtracting the last two equations from the first one and using Claim 1, we have

$$
(f(r, \mathbf{v})-f(r, \mathbf{0})-f(0, \mathbf{v})) f(0, \mathbf{v})=0 .
$$

If $\mathbf{v} \neq \mathbf{0}$, then multiply this equation from the right by the multiplicative inverse of $f(0, \mathbf{v})$ to get $f(r, \mathbf{v})=f(r, \mathbf{0})+f(0, \mathbf{v})$. It also holds for $\mathbf{v}=\mathbf{0}$ by Claim 1.

Claim 4: The solutions of (1) are precisely the functions given in the theorem.

By Claims 1, 2 and 3 we get the formula given in the theorem.

It can be checked by direct calculation that these are indeed solutions of (1).

\section{References}

[1] S. L. Altmann, Rotations, Quaternions, and Double Groups, Oxford University Press, 1986.

[2] A. Chronowski, On a certain type of Pexider equations, Ann. Math. Sil. 5 (1991), 75-82.

[3] W. Eichhorn, Über die multiplikative Cauchysche Funktionalgleichung für die Hamiltonschen Quaternionen und die Cayleyschen Zahlen, J. Reine Angew. Math. 221 (1966), 2-13. 
[4] T. A. Ell and S. J. Sangwine, Quaternion involutions and anti-involutions, Comput. Math. Appl. 53 (2007), 137-143.

[5] K. Heuvers and C. T. Ng, Multiplicative quadratic forms on the quaternions and related functional equations, Sci. Math. Jpn. 70 (2009), 29-41.

[6] I. L. Kantor and A. S. Solodovnikov, Hypercomplex Numbers, Springer-Verlag, 1989.

[7] D. Kobal and P. Šemrl, A result concerning additive maps on the set of quaternions and an application, Bull. Austral. Math. Soc. 44 (1991), 477-482.

[8] I. Makai, Cauchy-Gleichungen für Quaternionenfunktionen, Publ. Math. Debrecen 12 (1965), 253-258.

[9] J. Vince, Quaternions for Computer Graphics, Springer-Verlag, 2011.

[10] J. P. Ward, Quaternions and Cayley Numbers, Kluwer Academic Publishers, 1997.

Balázs Nyul

Faculty of Informatics

University of Debrecen

H-4010 Debrecen P.O.Box 12

Hungary

e-mail: nyul.balazs@inf .unideb.hu

Gábor Nyul

Institute of Mathematics

University of Debrecen

H-4010 Debrecen P.O.Box 12

Hungary

e-mail: gnyul@science.unideb.hu 